MATTERS ARISING

\title{
Reply to "A Thermodynamic assessment of the reported room-temperature chemical synthesis of $\mathrm{C}_{2}{ }^{\prime \prime}$
}

\author{
Kazunori Miyamoto (10 1,6凶 , Shodai Narita1,6, Yui Masumoto1, Takahiro Hashishin', Taisei Osawa1, \\ Mutsumi Kimura (1) 2,3, Masahito Ochiai ${ }^{4}$ \& Masanobu Uchiyama (1) $1,3,5 \times$
}

REPLYING To H. RZEPA. Nature Communications https://doi.org/10.1038/s41467-021-21433-8 (2021)

W e are writing in response to Rzepa's theoretical analysis of the generation of $\mathrm{C}_{2}$, which cites our recent paper describing the first chemical synthesis of $\mathrm{C}_{2}$ (ref. ${ }^{1}$ ). Rzepa suggests on the basis of several in silico approaches that the formation of free $C_{2}$ from alkynyl- $\lambda^{3}$ iodane and fluoride ion would be prohibitively endo-energetic. He proposes three possible explanations of the apparent discrepancy between these theoretical calculations and our experimental findings, of which one is that some species other than $\mathrm{C}_{2}$ is actually formed.

As our original paper was primarily experimental, describing room-temperature chemical synthesis of $\mathrm{C}_{2}$ and the first bottom-up chemical synthesis of nanocarbons from $\mathrm{C}_{2}$, we should like to respond to the latter point. We believe that the evidence presented in our paper for the generation of $\mathrm{C}_{2}$ itself is compelling ${ }^{1}$. In particular, the connected-flask, solvent-free experiment clearly supports the generation of free $C_{2}$ gas, for the following reasons:

1. When ${ }^{13} C$-labeled $\mathbf{1} \mathbf{b}-{ }^{13} C_{\beta}$ was used, a $1: 1$ mixture of $O$ ethynyl galvinoxyl $15^{-13} C_{\alpha}$ and $15^{13} C_{\beta}$ was obtained (Fig. 1a).

2. APCI mass spectrum of the contents in Flask B included the peak assignable to acetylene digalvinoxyl ether $\mathbf{1 6}$ (Fig. 1b).
It is more difficult to establish conclusively whether free $C_{2}$ is generated in solution, but the following experimental facts are relevant:

3. The relative rate of hydrogen abstraction between $\mathrm{CH}_{2} \mathrm{Cl}_{2}$ and 9,10-dihydroanthracene (12) is calculated to be ca. 1:20 (per 1H).

4. Galvinoxyl radical 14 solely produced $O$-ethynyl galvinoxyl 15 (not 16).

5. The amount of the mixture of $15-{ }^{13} C_{\alpha}$ and $15-{ }^{13} C_{\beta}$ obtained by the reaction of $\mathbf{1 b}-{ }^{13} \mathrm{C}_{\beta}$ with $\mathbf{1 4}$ differed in solvents of different viscosities $(\eta)$.

These results indicated that the radical character of the intermediate is much milder than that of the common unstabilized alkynyl radical, which is consistent with the presence of an interaction between the radicals in $\mathrm{C}_{2}$, in other words, a singlet biradical (charge-shift bonding) character, as suggested in a recent theoretical study ${ }^{2}$.

At present, we cannot explain the discrepancy between Rzepa's theoretical evaluation and our experimental results. However, as discussed in our original paper, we believe that all our experimental observations can only be rationalized in terms of the generation of gaseous $\mathrm{C}_{2}$. Our findings have attracted great interest, and we anticipate that independent experimental findings will emerge in the near future. We ourselves are

\footnotetext{
${ }^{1}$ Graduate School of Pharmaceutical Sciences, The University of Tokyo, Tokyo, Japan. ${ }^{2}$ Division of Chemistry and Materials, Faculty of Textile Science and Technology, Shinshu University, Ueda, Japan. ${ }^{3}$ Research Initiative for Supra-Materials (RISM), Shinshu University, Ueda, Japan. ${ }^{4}$ Graduate School of Pharmaceutical Sciences, University of Tokushima, Tokushima, Japan. ${ }^{5}$ Cluster of Pioneering Research (CPR), Advanced Elements Chemistry Laboratory, RIKEN, Saitama, Japan. ${ }^{6}$ These authors contributed equally: Kazunori Miyamoto, Shodai Narita. ${ }^{\circledR}$ email: kmiya@mol.f.u-tokyo.ac.jp; uchiyama@mol.f.u-tokyo.ac.jp
} 
a

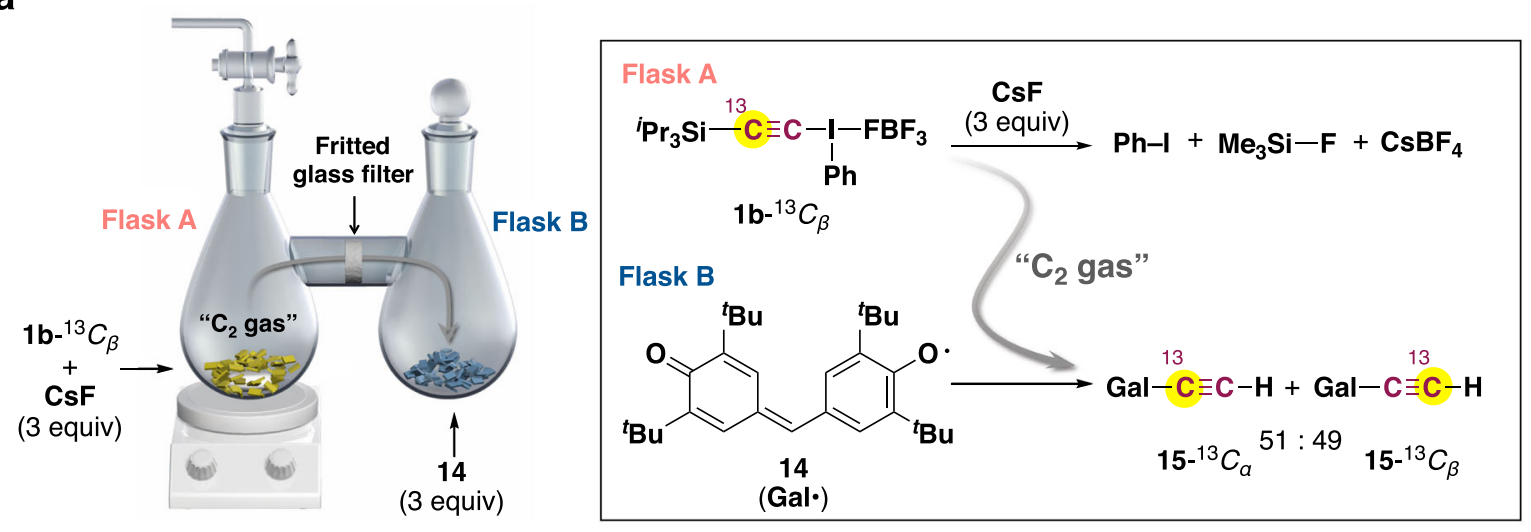

b

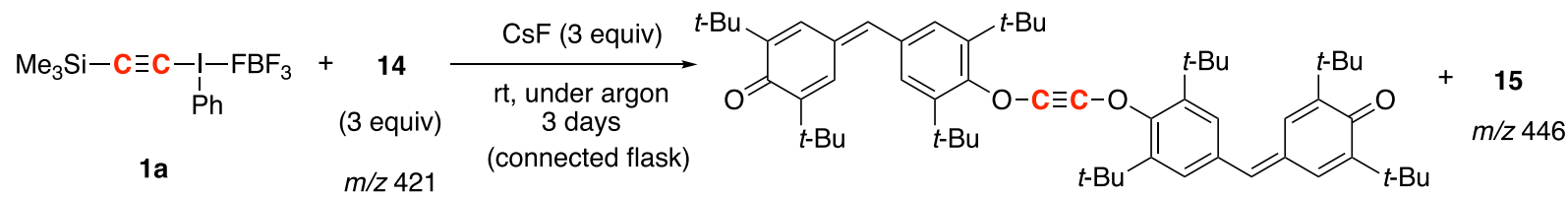

$16 \mathrm{~m} / \mathrm{z} 866$
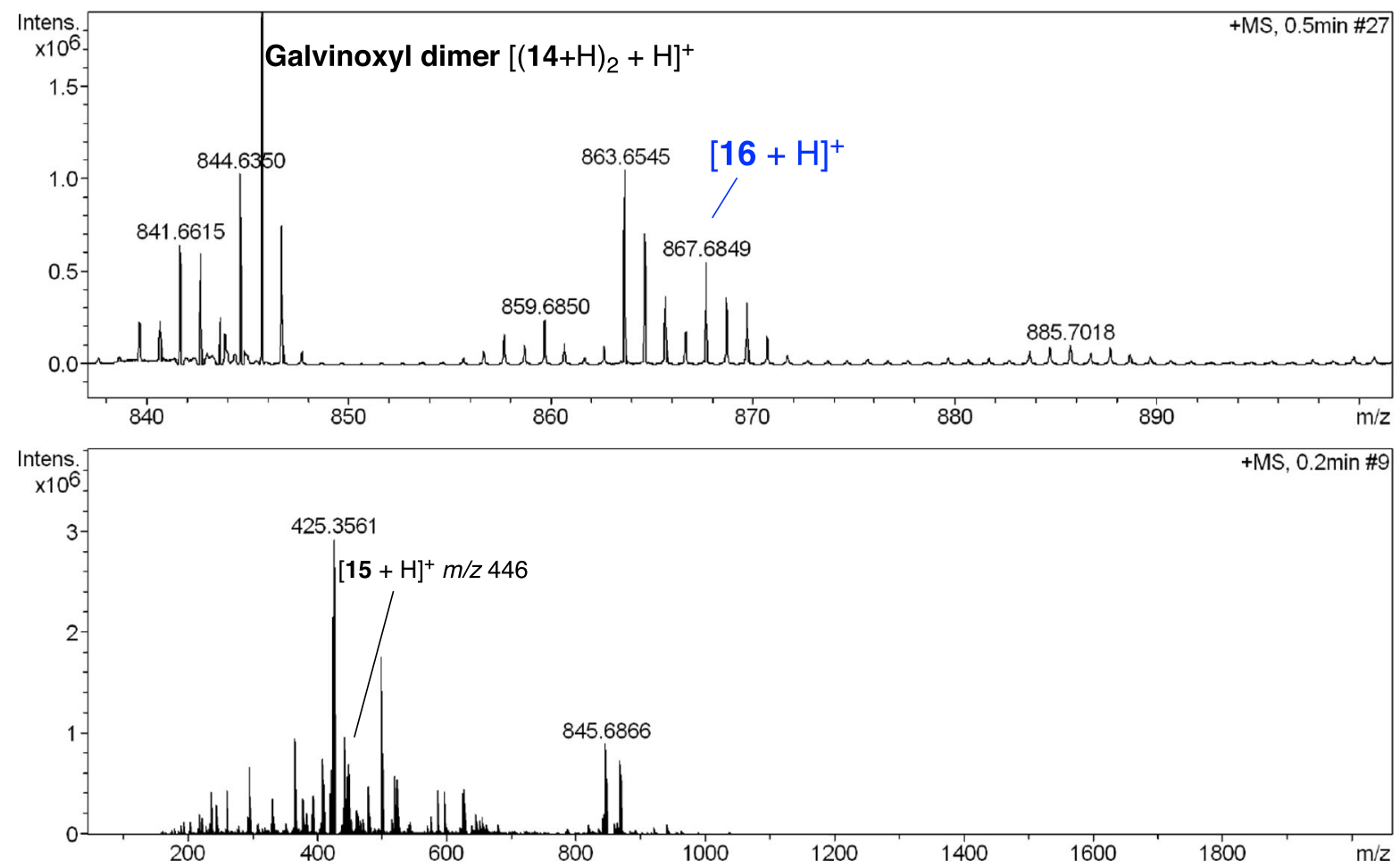

Fig. 1 Connected-flask, solvent-free experiment. a ${ }^{13} \mathrm{C}$-labeling experiment. $\mathbf{b} \mathrm{APCl}$ mass spectrum (positive ion mode) obtained from the contents in Flask B.

working on the direct observation of chemically generated $\mathrm{C}_{2}$ by means of Raman spectroscopy, ESR spectroscopy, and other methods, and we hope that this work will provide definitive experimental evidence for the bond length and electronic state of $\mathrm{C}_{2}$ (ref. ${ }^{3}$ and references therein).

\section{Data availability}

The data that support the findings of this study are available from the authors on request.
Received: 12 August 2020; Accepted: 19 January 2021; Published online: 23 February 2021

\section{References}

1. Miyamoto, K. et al. Room-temperature chemical synthesis of $\mathrm{C}_{2}$. Nat. Commun. 11, 2134 (2020). 
2. Shaik, S. et al. Charge-shift bonding: a new and unique form of bonding. Angew. Chem. Int. Ed. Engl. 59, 984-1001 (2020).

3. Shaik, S., Danovich, D., Braida, B. \& Hiberty, P. C. The quadruple bonding in $\mathrm{C}_{2}$ reproduces the properties of the molecule. Chem. Eur. J. 22, 4116 (2016).

\section{Author contributions}

K.M., M.O., and M.U. conceived and designed the experiments. S.N., Y.M., T.H., T.O., and K.M. conducted the experiments. K.M. and M.U. wrote the manuscript. All authors participated in data analyses and discussions. K.M., M.K., and M.U. directed the project.

\section{Competing interests}

The authors declare no competing interests.

\section{Additional information}

Correspondence and requests for materials should be addressed to K.M. or M.U.

Peer review information Nature Communications thanks the anonymous reviewers for their contribution to the peer review of this work.

Reprints and permission information is available at http://www.nature.com/reprints

Publisher's note Springer Nature remains neutral with regard to jurisdictional claims in published maps and institutional affiliations.

\section{cc) (i)}

Open Access This article is licensed under a Creative Common Attribution 4.0 International License, which permits use, sharing, adaptation, distribution and reproduction in any medium or format, as long as you give appropriate credit to the original author(s) and the source, provide a link to the Creative Commons license, and indicate if changes were made. The images or other third party material in this article are included in the article's Creative Commons license, unless indicated otherwise in a credit line to the material. If material is not included in the article's Creative Commons license and your intended use is not permitted by statutory regulation or exceeds the permitted use, you will need to obtain permission directly from the copyright holder. To view a copy of this license, visit http://creativecommons.org/ licenses/by/4.0/.

(C) The Author(s) 2021 